\title{
Effects of Gamma-Aminobutyric Acid Administration on Health and Growth Rate of Group-Housed Japanese Black Calves Fed Using an Automatic Controlled Milk Feeder
}

\author{
Daisaku MATSUMOTO ${ }^{1,2)}$, Mitsuhiro TAKAGI ${ }^{1) *}$, Yasuo FUSHIMI ${ }^{1)}$, Koji OKAMOTO ${ }^{3)}$, Mari KIDO $^{1)}$, \\ Miyo RYUNO ${ }^{1)}$, Yoshimi IMURA ${ }^{1)}$, Masanori MATSUNAGA ${ }^{4)}$, Koto INOKOSHI ${ }^{4)}$, Francis SHAHADA ${ }^{5)}$ and \\ Eisaburo DEGUCHI ${ }^{1)}$ \\ ${ }^{1)}$ Laboratories of Farm Animal Production Medicine and ${ }^{5)}$ Veterinary Public Health, Kagoshima University, Kagoshima 890-0064, \\ ${ }^{2)}$ Shepherd Central Livestock Clinic, Kagoshima 899-1611, ${ }^{3)}$ Soo Veterinary Clinical Center, Soo Agriculture Mutual Aid Association, \\ Kagoshima 899-8212 and ${ }^{4)}$ Nippon Zenyaku Kogyo Co., Ltd., Fukushima 963-0196, Japan
}

(Received 27 July 2008/Accepted 3 December 2008)

ABSTRACT. The efficacy of a gamma-aminobutyric acid (GABA) preparation in improving general health condition and growth rate was evaluated in suckling Japanese Black beef calves housed together and fed using an automatic milk feeder. The GABA preparation was administered from 4 to $10 \mathrm{~d}$ postpartum until weaning. A significant difference in mean body weight was observed at 3 and 8 weeks after introduction of the GABA preparation. The calves in the GABA group tended to require less medication than those in the control group ( $3.0 \mathrm{~d} /$ calf for BABA; $7.6 \mathrm{~d} /$ calf for the control; $\mathrm{P}=0.06$ ). The health statuses of the 2 groups may be reflected in their blood parameters. GABA may possibly have etiotropic effects in group-housed calves fed using an automatic milk feeder. KEY WORDS: automatic milk feeder system, calf, GABA, group housing.

Recently, group housing of preweaned calves has attracted increasing interest mainly because of concerns regarding calf welfare and labor efficiency. However, these systems are frequently set up for large groups, often comprising calves with a large age variation to which new young calves are continuously introduced (dynamic group) [12]. In addition, previous reports suggest that, compared with manual feeding, use of an automatic milk feeder for calves in group housing is associated with an increased risk of disease and reduced growth rate [9, 14]. Thus, the feeding regime affects the growth and general health conditions of young calves. Although conflicting results have been reported, it has been generally accepted that acute and chronic respiratory diseases and diarrhea occur more frequently in group housed veal calves fed using an automatic controlled milk feeding system than in individually reared calves. This might be due to the stress induced by group dynamics and difficulties with regard to maintaining the hygiene of the system [9].

The Japanese Black cattle is the most popular beef breed in Japan, and farmers are increasingly using automatic milk feeders in order to (1) promote the postpartum recovery of dam's estrous cycle, (2) achieve consistent growth of calves and (3) prevent diseases in calves. On the other hand, it is well recognized that, compared with other breeds, Japanese Black calves are relatively weak and, therefore, easily prone to sickness during the early postnatal period. This is probably due to immunodeficiencies in neonatal calves, such as

\footnotetext{
* CoRRespondence to: TAKAgi, M., Laboratory of Farm Animal Production Medicine, Kagoshima University, Kagoshima 8900064, Japan.

e-mail: mtakagi@agri.kagoshima-u.ac.jp
}

reduced lymphocyte proliferation $[6,10]$.

Gamma-aminobutyric acid (GABA) is an inhibitory neurotransmitter in the nervous system. It has been identified in many endocrine organs such as the pituitary, pancreas, gastrointestinal tract, ovary, placenta and adrenal medulla. This widespread distribution of GABA, its synthesizing and degrading enzymes and its receptors in the peripheral and central endocrine organs signify the involvement of GABA in regulation of endocrine functions [4]. A recent study indicated that mouse and human peripheral blood mononuclear cells, including lymphocytes, express functional $\mathrm{GABA}_{\mathrm{A}}$ receptors that probably modulate immune responses [2, 17]. Additionally, oral administration of GABA has been reported to alleviate several psychiatric disorders in humans and canines $[1,5,8]$. Based on these physiological effects, we speculated that the etiotropic effects of GABA could be applied to calves managed in dynamic group housing. On the other hand, in farm animal practice, particular attention has been paid to the impact of using antibiotics not only for preventing the emergence of drug resistance but also from the viewpoint of food safety and public health [13].

Therefore, in the present study, we aimed to investigate the effects of a GABA preparation administered orally as a supplement in milk replacer to group-housed calves via an automatic milk feeding system. We evaluated the health condition, body weight gain and other metabolic effects based on blood examinations in calves before their weaning period.

All procedures involving animal handling and treatment were approved by the Committee for Animal Use and Care of Kagoshima University, Japan (No. H19-agri-017). Two 
experiments were conducted to determine the effects of GABA. In Experiment 1, also referred to as the preliminary observational trial, Japanese Black calves born between April and May 2006 at the Kagoshima University Experimental Farm were used. Next, based on the results of the Experiment 1, Experiment 2 was conducted at the same farm using calves born between December 2006 and February 2007; in Experiment 2, additional observations, such as blood examination, were included and an extended evaluation was performed.

Calving occurred naturally on the pasture in all cases, and the calves were fed by their respective dams at least first $3 \mathrm{~d}$ after birth. The calves were separated from their dams within 9 days after calving. Health examination, measurement of body weight and blood sampling were conducted prior to introducing the calves to the automatic milk feeding system (Calf feeder; WestfaliaSurge GmBH, Germany) on the farm. Thus, all the introduced calves were considered to have similar levels of stress due to infection. New calves were continuously introduced into the existing group, and the age variation between newly introduced and older calves was $\leq 3$ weeks. Throughout the study, each experimental unit was an approximately 5-10 m areas and contained less than 10 calves.

Clinical observation, hematological analysis, serum total protein (TP) level and $\gamma$-glutamyltransferase (GGT) activity were measured on the day of blood sampling. The calves were considered clinically healthy and to have suckled enough colostrum after birth based on previously established standards; TP > $4.9 \mathrm{~g} / \mathrm{d} l$ and GGT $>300 \mathrm{U} / \mathrm{l}[11,16$, 18]. All the calves were injected with vitamin A solution (50,000 IU; Kasaki-Mitaka K.K., Tokyo, Japan) and vitamin E solution (100 IU; Nippon Zenyaku Kogyo Co., Ltd., Fukushima, Japan) when they were introduced into the group housing pen.

The calves were then randomly assigned to either the GABA group ( $n=8 ; 4$ males and 4 females) or control group ( $n=7 ; 4$ males and 3 females) in Experiment 1 or to the GABA group ( $\mathrm{n}=7 ; 6$ males and 1 female) or control group ( $n=7 ; 5$ males and 2 females) in Experiment 2. All the calves were raised as a single group and fed milk replacer via an automatic milk feeder twice a day. The calves in the GABA group received a GABA preparation (containing 0.01\% GABA, 0.04\% antioxidant and 99.95\% excipient; Nippon Zenyaku Kogyo Co., Ltd.). The GABA was produced by natural fermentation using a specific strain of lactic bacteria as reported in a previous report [7]. This preparation was added to the milk replacer at a rate of $5 \mathrm{~g} / \mathrm{d}$ (GABA volume, approximately $50 \mathrm{mg}$ ) by using an automatic measuring dispenser during the entire experimental periods. The dose of the GABA preparation was determined based on a previous report on human experiment in which a dose of $100 \mathrm{mg} /$ day was administered [1]. Fresh water and a calf starter (Total digestible nutrients $>75 \%$, Crude protein > 25\%; Calf Manna, Kyodo Shiryo Co., Ltd., Japan) supplemented with minerals and vitamins were provided ad libitum. The volume of milk replacer fed at the beginning of the experiment was $4 \mathrm{l} / \mathrm{d}$ and was gradually increased to a maximum of $6 \mathrm{l} / \mathrm{d}$ until 1 week before weaning. The volume of the milk replacer was then decreased to $3 \mathrm{l} / \mathrm{d}$ until weaning, regardless of the body weight and sex of the calves.

In Experiment 1, the body weights of the calves were measured both on the day on which they were introduced to the pen (d 0) and at 4 weeks of age in order to monitor the effects of GABA on growth performance by calculating daily gains in weight. Health condition, including appetite and fecal consistencies, were also monitored daily during the entire experimental period (approximately 4 weeks) by experienced staff. Additionally, a veterinarian routinely visited the herd at least twice a week in order to monitor the health conditions of the calves. In cases in which the veterinarian or staff detected signs of disease based on clinical criteria such as diarrhea (feces of gruel-like or watery consistency), fever (rectal temperature $>39.5^{\circ} \mathrm{C}$ ) and respiratory disease (severely increased respiratory sounds accompanied by fever and coughing or a grayish to yellowish nasal discharge) as reported previously [14], the clinical diagnoses were determined as enteritis, bronchitis or pneumonia. Medical treatment with mainly systematic antibiotic accompanied by supportive therapies were administered by the veterinarian based on the clinical signs and physical (body temperature and auscultation) and blood sample examinations. The medical treatment prescribed by the veterinarian was continued until the clinical signs disappeared. All medical treatments administered to individual calves were recorded.

In Experiment 2, the body weights of all the examined calves were measured on $\mathrm{d} 0$ and on a weekly basis until 4 weeks. Measurements were then obtained at 8 and 16 weeks of age, that is, after the calves were introduced to the automatic milk feeding system. Additionally, blood samples from the jugular vein were collected on a weekly basis starting from d 0 until 4 weeks and at 8 weeks after being introduced into the pen in order to determine the complete blood count (CBC; F-820; Sysmex, Japan) and levels of serum aspartate aminotransferase (AST), blood urea nitrogen (BUN), total cholesterol (T-Cho), glucose (Glu), free fatty acid (FFA), triglyceride (TG), sialic acid (SA; Labospect 7080 autoanalyzer; Hitachi, Japan), haptoglobin (HP) and $\alpha 1$-acid glycoprotein ( $\alpha 1$-AGP; single-immunodiffusion method; Metabolic-Eco-System Institute, Japan), IgG and IgM (radial immunodiffusion method; Bovine IgG and IgM ELISA Quantitation Kit, Bethyl Laboratories, Inc., TX, U.S.A.), and vitamins A and E (VA and VE, respectively; high-performance light chromatography method). These tests were performed to monitor hepatic and renal function, nutritional status, inflammation and immune status from the day of introduction to the pen (d 0). Further more, all health checkups and medical treatments for the examined calves were conducted as described in Experiment 1.

The results of each group are expressed as means \pm SEM. Body weight, daily gain in weight, blood examination parameters, and mean days of medical treatment were compared between the 2 groups using the Student's $t$-test in 

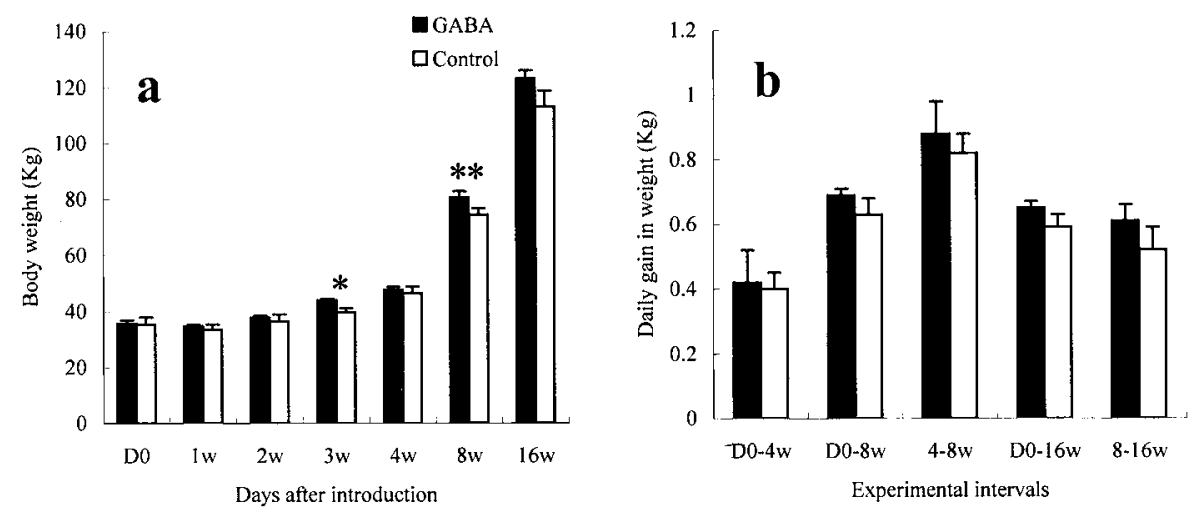

Fig. 1. Mean ( \pm SEM) body weights (a) and daily gains in weight (b) of the calves in the GABA and control groups. D 0: day of introduction to the pen. W: week after introduction. *: Significantly different between the GABA and control groups $(\mathrm{P}<0.05)$. **: Tended to be significantly different between the GABA and control groups $(\mathrm{P}=0.09)$.

order to determine the effects of GABA supplementation. P values less than 0.05 were considered to indicate a statistically significant difference, while $\mathrm{P}$ values less than 0.1 were considered to indicate a significant tendency.

In Experiment 1, the body weights of the calves in both the GABA (38.3 kg) and control (39.9 kg) groups did not differ significantly at the beginning of the experiment. The mean ( \pm SEM) daily gain in body weight during the experimental period (approximately 4 weeks) in the GABA group $(0.3 \pm 0.1 \mathrm{~kg})$ also did not differ significantly from that in the control group $(0.2 \pm 0.1 \mathrm{~kg})$. In the GABA group, medical treatment was provided for a total of 46 of the $228 \mathrm{~d}$ of automatic feeding, and the mean $( \pm$ SEM) number of days of medical treatment per calf was $5.8 \pm 1.1 \mathrm{~d}$; the respective numbers in the control group were 68 of the $198 \mathrm{~d}$ of automatic feeding and $9.7 \pm 2.4 \mathrm{~d}$. Although no significant difference $(\mathrm{P}=0.15)$ was observed in these numbers between the 2 groups, the total duration of medical treatment was lower in the GABA group than in the control group.

The mean body weight and daily gain in body weight recorded in Experiment 2 are shown in Fig. 1. At the beginning of the experiment, no significant differences in these parameters were observed between the GABA (35.7 kg) and control (35.3 kg) groups. During the experimental period, the mean body weight of the calves in the GABA group was significantly greater than that of the calves in the control group at 3 weeks (43.9 kg for GABA; $39.7 \mathrm{~kg}$ for the control; $\mathrm{P}=0.04$ ) and tended to be heavier at 8 weeks, i.e., at weaning (80.5 kg for GABA; $74.3 \mathrm{~kg}$ for the control; $\mathrm{P}=0.09$; Fig. 1a). However, there were no differences at 0 , 1,2 and 16 weeks. No significant difference was observed in mean daily gain in body weight at each time point between the GABA and control groups (Fig. 1b). Table 1 lists the total durations and mean numbers of days of medical treatment noted in the experiment. The total durations of medical treatment in the GABA and control groups were 21 out of the $456 \mathrm{~d}$ of automatic feeding and 53 out of the 425 $\mathrm{d}$ of automatic feeding, respectively; the total duration was
Table 1. Mean ( \pm SEM) number of medical treatment days and frequency (\%) of diseases in calves in the GABA and control groups during the 8-week study period

\begin{tabular}{ccc}
\hline & $\begin{array}{c}\text { GABA } \\
(\mathrm{n}=7)\end{array}$ & $\begin{array}{c}\text { Control } \\
(\mathrm{n}=7)\end{array}$ \\
\hline $\begin{array}{c}\text { Total days of the } \\
\text { experiment }\end{array}$ & 456 & 425 \\
$\begin{array}{c}\text { Duration of medical } \\
\text { treatment }(\%)\end{array}$ & $4.6^{\mathrm{a})}$ & $125^{\mathrm{a})}$ \\
$\begin{array}{c}\text { Mean number of medical } \\
\text { treatment days per calf }\end{array}$ & $3.0 \pm 1.2^{\mathrm{b})}$ & $7.6 \pm 1.8^{\mathrm{b})}$ \\
\hline
\end{tabular}

a) $\mathrm{P}<0.05$. b) $\mathrm{P}=0.06$.

significantly lower in the GABA group than in the control group $(\mathrm{P}<0.05)$. In addition, the mean number of days of medical treatment per calf in the GABA treatment group tended to be lower than that in the control group (3.0 $\mathrm{d}$ for GABA; $7.6 \mathrm{~d}$ for the control; $\mathrm{P}=0.06$ ). The diseases noted in the calves during the experimental period mainly involved the respiratory (bronchitis and pneumonia) and digestive (diarrhea) systems. In the GABA treatment group, $60.7 \%$ of diseases were attributed to respiratory infections, whereas $39.3 \%$ of diseases were attributed to digestive disorders. On the other hand, in the control group, $29.5 \%$ of diseases were respiratory diseases, and the remaining $68.9 \%$ of diseases were digestive diseases.

The results of hematological and serum biochemical analyses (TP and GGT level) are shown in Fig. 2. No significant differences were observed between the GABA and control groups with regard to the red blood cell, white blood cell, hemoglobin and hematocrit values during the entire study period. Based on the TP (> $4.9 \mathrm{~g} / \mathrm{d} l)$ and GGT (> $300 \mathrm{U} / \mathrm{l})$ concentrations measured on the day the calves were introduced into the pen, all the examined calves from both the groups were considered to have suckled enough colostrum from their dams immediately after calving. Although the ranges of these parameters were within the normal limits in 

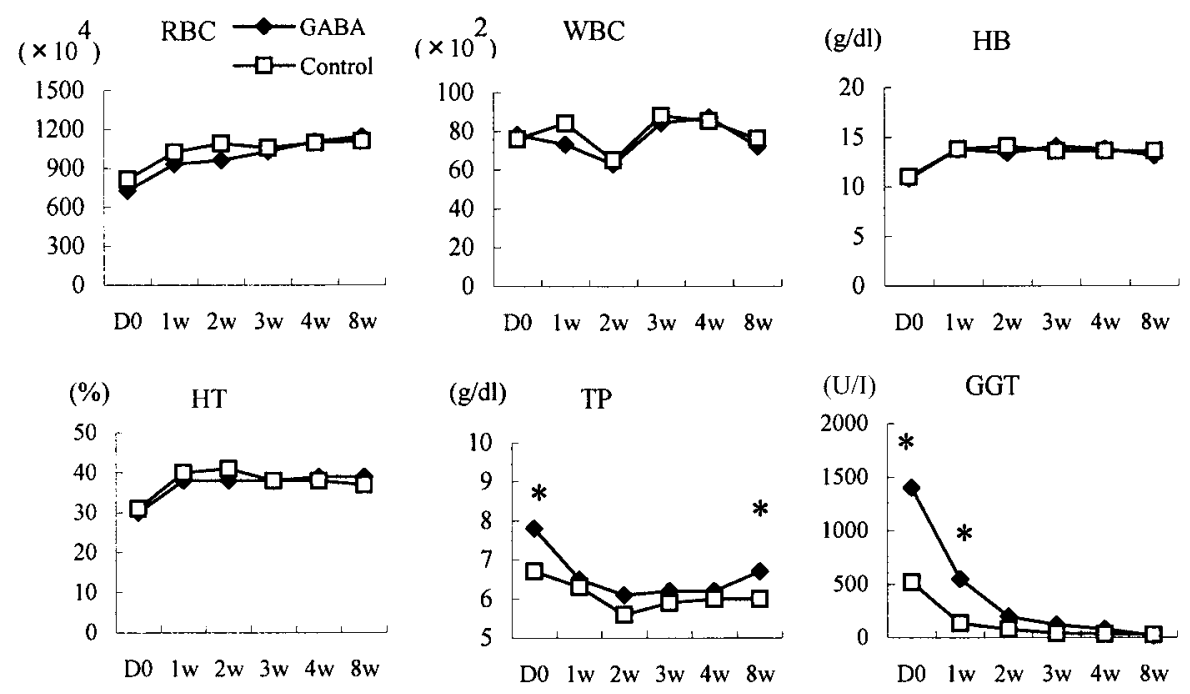

Fig. 2. Results of hematological and serum biochemical analyses. Blood samples were obtained on d 0 (day of introduction to the feeder system). The analysis involved measurements of the red blood cell (RBC) and white blood cell (WBC) counts and levels of hemoglobin (HB), hematocrit (HT), total protein (TP) and $\gamma$-glutamyltransferase (GGT). These parameters were used for monitoring the colostrum suckling status. D 0: day of introduction to the pen W: week after introduction. *: Significantly different between the GABA and control groups $(\mathrm{P}<0.05)$.

both groups, the TP concentration in the GABA group was significantly higher than that in the control group on the day of introduction (7.8 g/d l for GABA; $6.7 \mathrm{~g} / \mathrm{d} l$ for the control; $\mathrm{P}<0.05)$ and at 8 weeks after introduction $(6.7 \mathrm{~g} / \mathrm{d} l$ for GABA; $6.0 \mathrm{~g} / \mathrm{d} l$ for the control; $\mathrm{P}<0.05)$. Similarly, a significant difference in the GGT concentration was observed between the GABA (1399 U/l at d 0; $546 \mathrm{U} / \mathrm{l}$ at 1 week:) and control (515 $\mathrm{U} / \mathrm{l}$ at $\mathrm{d} 0 ; 135 \mathrm{U} / \mathrm{l}$ at 1 week; $\mathrm{P}<0.05)$ groups until 1 week after introduction of the calves into the pen.

The results of serum biochemical analyses are shown in Fig. 3. No significant differences were observed in the levels of AST, BUN, Glu, FFA, $\alpha 1$-AGP, SA and VA between the GABA and control groups during the entire study period. Significant differences between the GABA and control groups were observed in the T-Cho concentration at 1 week (66 mg/dl for GABA; $44 \mathrm{mg} / \mathrm{d} l$ for the control) and 2 weeks ( $94 \mathrm{mg} / \mathrm{d} l$ for GABA; $59 \mathrm{mg} / \mathrm{d} l$ for the control) after introduction, in the TG concentration at 1 week after introduction (18 mg/d $l$ for GABA; $7 \mathrm{mg} / \mathrm{d} l$ for the control), in the VE concentration at 1 week $(1.0 \mu \mathrm{g} / \mathrm{ml}$ for GABA; $0.4 \mu \mathrm{g} /$ $\mathrm{ml}$ for the control) and 2 weeks after introduction $(1.6 \mu \mathrm{g} / \mathrm{ml}$ for GABA; $0.6 \mu \mathrm{g} / \mathrm{ml}$ for the control), in the IgG concentration at $\mathrm{d} 0(14.8 \mathrm{mg} / \mathrm{ml}$ for GABA; $10.2 \mathrm{mg} / \mathrm{ml}$ for the control) and 4 weeks after introduction $(10.8 \mathrm{mg} / \mathrm{ml}$ for GABA; $6.9 \mathrm{mg} / \mathrm{ml}$ for the control) and in the IgM concentration at $\mathrm{d}$ 0 (10.7 $\mathrm{mg} / \mathrm{ml}$ for GABA; $7.4 \mathrm{mg} / \mathrm{ml}$ for the control). The number of HP positive calves was not statistically different between the 2 groups at 1 week ( 3 for GABA; 2 for the control), 2 weeks ( 0 for GABA; 1 for the control), 3 weeks (2 for GABA; 0 for the control), 4 weeks ( 3 for GABA; 2 for the control), and 8 weeks ( 0 for GABA; 1 for the control).
It has been suggested that infectious respiratory and digestive diseases may be more frequent in group housed beef calves than in those maintained in crates [9], probably due to a large group and continuous introduction of new calves [12], which may experience a stressful environment. The reduced total duration of medical treatments in the GABA group suggests that administration of a GABA preparation mixed in with milk replacer to calves before the weaning period may have etiotropic effects on the health status of calves, and hence, on their growth rates.

In the present study, we observed that GABA administration before weaning had etiotropic effects on the health statuses and growth rates of the calves. Additionally, we demonstrated a significant reduction in (1) the duration of medical treatment of sick calves, (2) the number of calves diagnosed with diarrhea and (3) the number of calves that required medical treatment for more than 2 days. These results could imply that enteric inflammation in the calves in the GABA group was not severe and that the GABA-treated calves experienced faster recovery from illnesses than the control calves. The results of the blood examinations also reflected the health condition in both the groups. The T-Cho concentration was found to be significantly lower in the control group than in the GABA group during the first and second weeks after introduction of the calves into the pen. This may be due to restriction of the enterohepatic circulation by both severe diarrhea and impaired intestinal mucosa and to reduced absorption of lipids from the feeds. Additionally, the serum biochemical profiles of the control calves showed low glucose and TG levels but a high FFA concentration. This may reflect negative energy balance 

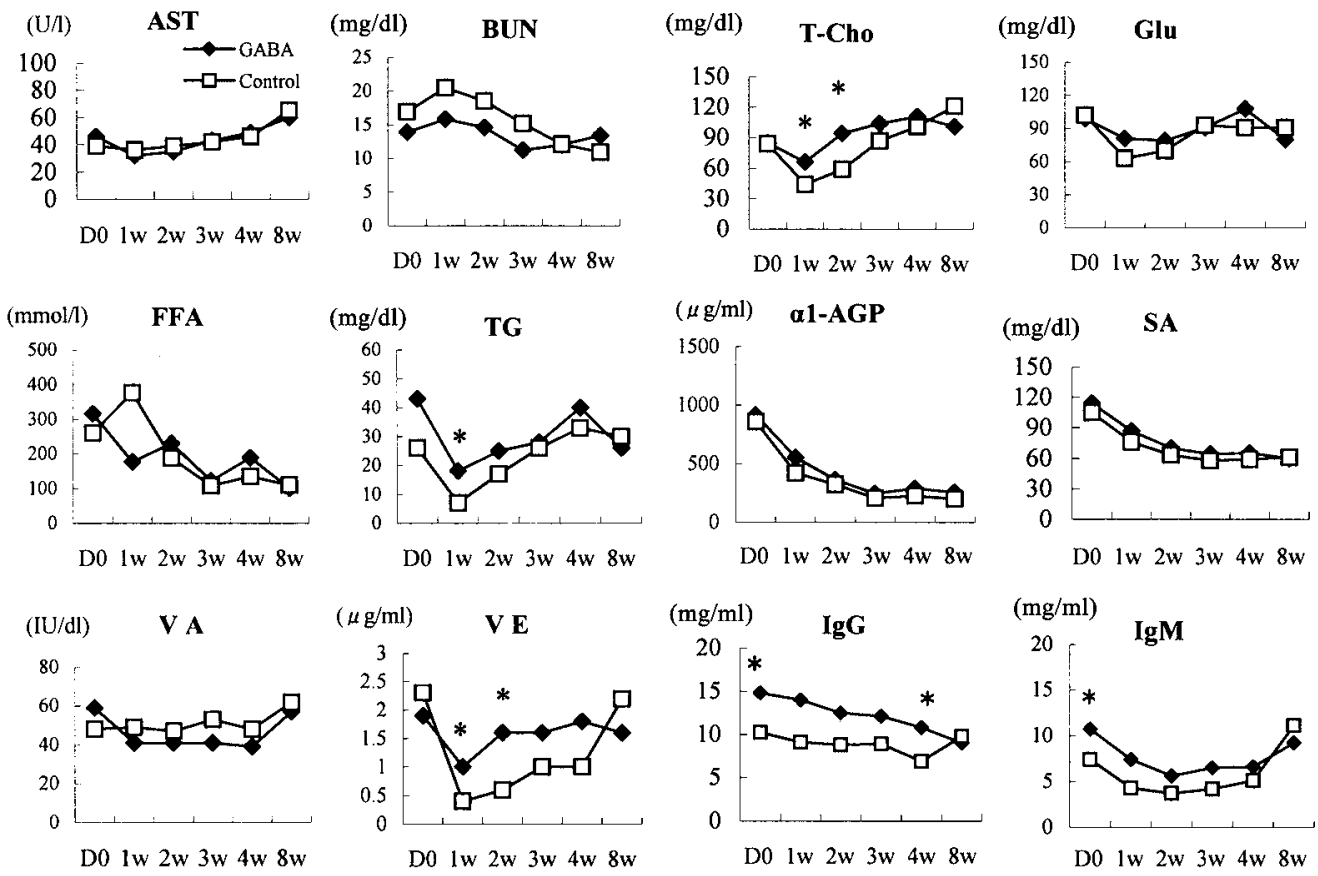

Fig. 3. Results of serum biological analyses after introduction to the pen. Hepatic function was assessed by determining the levels of aspartate aminotransferase (AST); renal function was assessed by determining the blood urea nitrogen (BUN) level; nutritional condition was assessed by determining the levels of total cholesterol (TCho), glucose (Glu), free fatty acid (FFA) and triglyceride (TG); inflammation status was assessed by determining the levels of $\alpha 1$-acid glycoprotein ( $\alpha 1$-AGP) and sialic acid (SA); vitamin status was assessed by determining the levels of vitamin A (VA) and vitamin E (VE) and immuno status was assessed by determining the level of IgG and IgM.D 0: the day of introduction. W: week after introduction. *: Significantly different between the GABA and control groups $(\mathrm{P}<0.05)$.

during the critical (medication) period and may support the above-mentioned assumption of impaired nutritional absorption, which has been previously noted in Japanese Black cattle with growth retardation [15]. Moreover, the concentration of VE in the GABA group was significantly higher than that in the control group during the first and second weeks, although the calves in both groups were administered equal units of $\mathrm{VE}$ at the beginning of the experiment. This could be attributed to a difference in health status between the 2 groups, in which the consumption of VE in the GABA group was possibly lower than that of control group.

When the calves were introduced to the automatic milk feeding system, significant differences in both the IgG and IgM concentrations were observed between the 2 groups on d 0 in Experiment 2. Because the calves were separated from their dams between 3 and 9 days after calving, these differences in the IgG and IgM concentrations might be due to differences on the day of introduction of each calf into the experimental pen after calving. It has been reported that calves with a serum IgG concentration of less than $10 \mathrm{mg} / \mathrm{ml}$ have higher mortality [3] and that $8.0 \mathrm{mg} / \mathrm{ml}$ is the marginal IgG concentration at $24 \mathrm{hr}$ after calving [19]. We measured the concentrations of serum GGT and TP in all the calves in the GABA and control groups, and the findings suggest that the individual calves consumed enough colostrum immediately after calving. Thus, we assumed that all the calves from both groups possessed adequate ability to prevent infectious enteric and respiratory diseases from the beginning of the experiment. However, it has been reported that the passive immune status of a calf during the 24-hr postpartum period is an important determinant of health before and after weaning and is indirectly associated with calf growth during this period $[3,19]$. Therefore, further investigation is required to clarify whether the observed difference in health status between the 2 groups was derived from the etiotropic effect of GABA or the amount of colostrum consumed within 24-hr postpartum.

Based on the results of daily clinical observations, blood examinations, and daily gain in body weight, the GABA preparation can be considered to have comparatively less side effects, particularly on the hepatic, renal and general metabolic functions, and is likely a safe daily supplement. Thus, our findings provide insights into the new strategy of using a GABA preparation for preventing disease in group housed beef calves maintained using an automated milk feeding system. 


\section{REFERENCES}

1. Abdou, A.M., Higashiguchi, S., Horie, K., Kim, M., Hatta, H. and Yokogoshi, H. 2006. Relaxation and immunity enhancement effect of $\gamma$-Aminobutyric acid (GABA) administration in humans. Bio. Factors. 26: 201-208.

2. Alam, S., Laughton, D.L., Walding, A. and Wolstenholme, A.J. 2006. Human peripheral blood mononuclear cells express GABAA receptor subunits. Mol. Immunol. 43: 1432-1442.

3. Besser, T.E. and Gay, C.C. 1994. The importance of colostrum to the health of the neonatal calf. Vet. Clin. North Am. Food Anim. Pract. 10: 107-117.

4. Gladkevich A., Korf, J., Hakobyan, V.P. and Melkonyan, K.V. 2006. The peripheral GABAergic system as a target in endocrine disorders. Auton. Neurosci-Basic. 124: 1-8.

5. Inagawa, K., Seki, S., Bannai, M., Takeuchi, Y., Mori, Y. and Takahashi, M. 2005. Alleviative effects of $\gamma$-aminobutyric acid (GABA) on behavioral abnormalities in aged dogs. J. Vet. Med. Sci. 67: 1063-1066.

6. Inokuma, H., Yoshida, T. and Onishi, T. 1995. Development of peripheral blood mononuclear cell response to mitogens in Japanese Black newborn calves. J. Vet. Med. Sci. 57: 971-972.

7. Komatsuzaki, N., Shima, J., Kawamoto, S., Momose, H. and Kimura, T. 2005. Production of $\gamma$-aminobutyric acid (GABA) by Lactobacillus paracasei isolated from traditional fermented foods. Food Microbiol. 22: 497-504.

8. Krystal, J.H., Sanacora, G., Blumberg, H., Anand, A., Charney, D.S., Marek, G., Epperson, C.N., Goddard, A. and Mason, G.F. 2002. Glutamate and GABA system as targets for novel antidepressant and mood-stabilizing treatments. Mol. Psychiatry 7: 71-80.

9. Maatje, K., Verhoeff, J., Kremer, W.D., Cruijsen, A.L. and van den Ingh, T.S. 1993. Automated feeding of milk replacer and health control of group-housed veal calves. Vet. Rec. 133: 266270.

10. Ohtsuka, H., Komatsu, S., Kon-nai, S., Fukuda, S., Kuku, Y.,
Yoshino, T., Koiwa, M. and Kawamura, S. 2002. Comparison of peripheral leukocytes in Japanese Black and Holstein calves. J. Jpn. Vet. Med. Assoc. 55: 789-795 (in Japanese, with English abstract).

11. Perino, L.J., Sutberland, R.L. and Woollen, N.E. 1993. Serum $\gamma$-glutamyltransferase activity and protein concentration at birth and after suckling in calves with adequate and inadequate passive transfer of immunoglobulin G. Am. J. Vet. Res. 54: 5659.

12. Rasmussen, L., Jensen, M.B. and Jeppesen, L.L. 2006. The effect of age at introduction and number of milk-portions on the behavior of group housed dairy calves fed by a computer controlled milk feeder. Appl. Anim. Behav. Sci. 100: 153-163.

13. Refsdal, A.O. 2000. To treat or not treat: a proper use of hormones and antibiotics. Anim. Reprod. Sci. 60-61: 109-119.

14. Svensson, C. and Liberg, P. 2006. The effect of group size on health and growth rate of Swedish dairy calves housed in pens with automatic milk-feeders. Prev. Vet. Med. 73: 43-53.

15. Takasu, M., Yayota, M., Nakano, M., Nishii, N., Ohba, Y., Okada, K., Maeda, S., Miyazawa, K. and Kitagawa, H. 2005. Results of metabolic profile test in Japanese Black cattle with growth retardation. J. Vet. Med. Sci. 67: 1269-1271.

16. Thompson, J.C. and Pauli, J.V. 1981. Colostral transfer of gamma glutamyl transpeptidase in calves. $N$. Z. Vet. J. 29: 223-226.

17. Tian, J., Lu, Y.L., Zhang, H., Chau, C.H., Dang, H.N. and Kaufman, D.L. 2004. $\gamma$-Aminobutyric acid inhibits T cell autoimmunity and the development of inflammatory responses in a mouse type 1 diabetes model. J. Immunol. 173: 52985304.

18. Wesselink, R., Stafford, K.J., Mellor, D.J., Todd, S. and Gregory, N.G. 1999. Colostrum intake by dairy calves. N. Z. Vet. J. 47: 31-34.

19. Wittum, T.E. and Perino, L.J. 1995. Passive immune status at postpartum hour 24 and long-term health and performance of calves. Am. J. Vet. Res. 56: 1149-1154. 